\title{
A Chemical Sensor For The Liquid-Ordered Phase
}

Honghua Cao, Jianbing Zhang, Bingwen Jing and Steven L. Regen*

Department of Chemistry, Lehigh University, Bethlehem, Pennsylvania 18015

Supporting Information 
Table 1. 40\% Cholesterol + DPPC

\begin{tabular}{|c|c|c|c|}
\hline \multirow{2}{*}{} & \multicolumn{3}{|c|}{ Mol \% of equilibrium product mixtures } \\
\cline { 2 - 4 } & \multicolumn{3}{|c|}{ Starting from $\{\mathbf{1 , 1}\}$ and $\{\mathbf{2 , 2}\}$} \\
\hline Time, hrs & $\mathbf{1 1 , 1}\}$ & $\mathbf{1 , 2}\}$ & $\{\mathbf{2 , 2}\}$ \\
\hline 1 & 24.96 & 50.75 & 24.29 \\
\hline 2 & 23.30 & 54.47 & 22.23 \\
\hline 3 & 23.44 & 54.02 & 22.53 \\
\hline 4 & 23.69 & 53.91 & 22.40 \\
\hline 5 & 23.84 & 53.87 & 22.28 \\
\hline 6 & 23.49 & 53.39 & 23.13 \\
\hline \multicolumn{3}{|c|}{ Starting from $\mathbf{1 1 , 2}\}$} \\
\hline 1 & 23.16 & 54.13 & 22.71 \\
\hline 2 & 22.29 & 53.43 & 24.28 \\
\hline 3 & 23.03 & 53.85 & 23.11 \\
\hline 4 & 21.79 & 53.90 & 24.31 \\
\hline 5 & 23.19 & 53.58 & 23.23 \\
\hline 6 & 22.20 & 53.10 & 24.69 \\
\hline
\end{tabular}

Table 2. $35 \%$ Cholesterol + DPPC

\begin{tabular}{|c|c|c|c|}
\hline \multirow{2}{*}{} & \multicolumn{3}{|c|}{ Mol \% of equilibrium product mixtures } \\
\cline { 2 - 4 } & \multicolumn{3}{|c|}{ Starting from $\{\mathbf{1 , 1}\}$ and $\mathbf{2}, \mathbf{2}\}$} \\
\hline Time, hrs & $\mathbf{1 , 1}\}$ & $\{\mathbf{1 , 2}\}$ & $\{\mathbf{2 , 2}\}$ \\
\hline 1 & 21.00 & 54.28 & 24.72 \\
\hline 2 & 20.97 & 54.13 & 24.90 \\
\hline 3 & 21.91 & 53.59 & 24.50 \\
\hline 4 & 21.97 & 53.79 & 24.24 \\
\hline 5 & 22.98 & 53.90 & 23.12 \\
\hline 6 & 23.19 & 53.45 & 23.36 \\
\hline \multicolumn{3}{|c|}{ Starting from $\mathbf{1 1 , 2}\}$} \\
\hline 1 & 21.23 & 54.07 & 24.70 \\
\hline 2 & 19.98 & 54.07 & 25.95 \\
\hline 3 & 20.58 & 53.97 & 25.46 \\
\hline 4 & 22.34 & 52.27 & 25.38 \\
\hline 5 & 22.33 & 53.96 & 23.70 \\
\hline 6 & 21.65 & 53.70 & 24.65 \\
\hline
\end{tabular}


Table 3. $30 \%$ Cholesterol + DPPC

\begin{tabular}{|c|c|c|c|}
\hline \multirow{2}{*}{} & \multicolumn{3}{|c|}{ Mol \% of equilibrium product mixtures } \\
\cline { 2 - 4 } & \multicolumn{3}{|c|}{ Starting from $\{\mathbf{1 , 1}\}$ and $\{\mathbf{2 , 2}\}$} \\
\hline Time, hrs & $\mathbf{1 1 , 1}\}$ & $\mathbf{1 1 , 2}\}$ & $\{\mathbf{2 , 2}\}$ \\
\hline 1 & 23.20 & 54.17 & 22.63 \\
\hline 2 & 23.28 & 54.21 & 22.51 \\
\hline 3 & 23.24 & 54.20 & 22.55 \\
\hline 4 & 23.95 & 53.96 & 22.09 \\
\hline 5 & 22.52 & 54.35 & 23.13 \\
\hline 6 & 23.13 & 53.99 & 22.88 \\
\hline & \multicolumn{3}{|c|}{ Starting from $\{\mathbf{1 , 2}\}$} \\
\hline 1 & 15.91 & 64.86 & 19.23 \\
\hline 2 & 21.34 & 54.10 & 24.55 \\
\hline 3 & 20.92 & 54.10 & 24.97 \\
\hline 4 & 20.54 & 54.44 & 25.02 \\
\hline 5 & 21.96 & 54.14 & 23.89 \\
\hline 6 & 21.42 & 54.12 & 24.46 \\
\hline
\end{tabular}

Table 4. 27.5\% Cholesterol + DPPC

\begin{tabular}{|c|c|c|c|}
\hline \multirow{2}{*}{} & \multicolumn{3}{|c|}{ Mol \% of equilibrium product mixtures } \\
\cline { 2 - 4 } & \multicolumn{3}{|c|}{ Starting from $\{\mathbf{1 , 1}\}$ and $\{\mathbf{2 , 2}\}$} \\
\hline Time, hrs & $\mathbf{1 1 , 1}\}$ & $\{\mathbf{1 , 2}\}$ & $\{\mathbf{2 , 2}\}$ \\
\hline 1 & 22.45 & 53.85 & 23.69 \\
\hline 2 & 25.71 & 52.15 & 22.14 \\
\hline 3 & 25.06 & 53.18 & 21.76 \\
\hline 4 & 22.98 & 53.00 & 24.02 \\
\hline 5 & 25.90 & 52.68 & 21.42 \\
\hline 6 & 22.45 & 52.38 & 25.16 \\
\hline & \multicolumn{3}{|c|}{ Starting from $\{\mathbf{1 , 2}\}$} \\
\hline 1 & 23.13 & 53.02 & 23.86 \\
\hline 2 & 22.98 & 53.16 & 23.86 \\
\hline 3 & 23.11 & 53.27 & 23.62 \\
\hline 4 & 22.74 & 53.00 & 24.26 \\
\hline 5 & 23.48 & 53.52 & 23.00 \\
\hline 6 & 21.74 & 53.35 & 24.91 \\
\hline
\end{tabular}


Table 5. 25\% Cholesterol + DPPC

\begin{tabular}{|c|c|c|c|}
\hline \multirow{2}{*}{} & \multicolumn{3}{|c|}{ Mol \% of equilibrium product mixtures } \\
\cline { 2 - 4 } & \multicolumn{3}{|c|}{ Starting from $\{\mathbf{1 , 1}\}$ and $\{\mathbf{2 , 2}\}$} \\
\hline Time, hrs & $\mathbf{1 1 , 1}\}$ & $\mathbf{1 , 2}\}$ & $\{\mathbf{2 , 2}\}$ \\
\hline 1 & 21.51 & 52.63 & 25.86 \\
\hline 2 & 22.16 & 53.29 & 24.55 \\
\hline 3 & 22.91 & 52.75 & 24.34 \\
\hline 4 & 22.62 & 53.03 & 24.35 \\
\hline 5 & 22.42 & 52.52 & 25.06 \\
\hline 6 & 21.63 & 53.27 & 25.10 \\
\hline \multicolumn{3}{|c|}{ Starting from $\mathbf{1 1 , 2}\}$} \\
\hline 1 & 21.39 & 52.72 & 25.90 \\
\hline 2 & 22.46 & 52.92 & 24.62 \\
\hline 3 & 21.46 & 52.36 & 26.18 \\
\hline 4 & 21.65 & 52.25 & 26.10 \\
\hline 5 & 23.11 & 52.84 & 24.05 \\
\hline 6 & 20.68 & 52.64 & 26.68 \\
\hline
\end{tabular}

Table 6. $22.5 \%$ Cholesterol + Cholesterol

\begin{tabular}{|c|c|c|c|}
\hline \multirow{2}{*}{} & \multicolumn{3}{|c|}{ Mol \% of equilibrium product mixtures } \\
\cline { 2 - 4 } & \multicolumn{3}{|c|}{ Starting from $\{\mathbf{1 , 1}\}$ and $\{\mathbf{2 , 2}\}$} \\
\hline Time, hrs & $\mathbf{1 , 1}\}$ & $\mathbf{1 , 2}\}$ & $\{\mathbf{2 , 2}\}$ \\
\hline 1 & 23.71 & 52.52 & 23.77 \\
\hline 2 & 23.68 & 51.30 & 25.03 \\
\hline 3 & 25.56 & 51.48 & 22.96 \\
\hline 4 & 25.17 & 51.42 & 23.41 \\
\hline 5 & 23.99 & 51.18 & 24.84 \\
\hline 6 & 23.56 & 51.01 & 25.43 \\
\hline \multicolumn{3}{|c|}{ Starting from $\mathbf{1 1 , 2}\}$} \\
\hline 1 & 20.87 & 52.22 & 26.91 \\
\hline 2 & 21.96 & 52.47 & 25.57 \\
\hline 3 & 20.13 & 52.55 & 27.32 \\
\hline 4 & 20.35 & 52.28 & 27.37 \\
\hline 5 & 20.93 & 51.35 & 27.72 \\
\hline 6 & 21.34 & 52.17 & 26.48 \\
\hline
\end{tabular}


Table 7. 20\% Cholesterol + DPPC

\begin{tabular}{|c|c|c|c|}
\hline \multirow{2}{*}{} & \multicolumn{3}{|c|}{ Mol \% of equilibrium product mixtures } \\
\cline { 2 - 4 } & \multicolumn{3}{|c|}{ Starting from $\{\mathbf{1 , 1}\}$ and $\{\mathbf{2 , 2}\}$} \\
\hline Time, hrs & $\{\mathbf{1 , 1}\}$ & $\mathbf{1 1 , 2}\}$ & $\{\mathbf{2 , 2}\}$ \\
\hline 2 & 22.46 & 51.02 & 26.52 \\
\hline 3 & 20.72 & 50.16 & 29.12 \\
\hline 4 & 23.50 & 50.40 & 26.10 \\
\hline 5 & 22.12 & 50.17 & 27.71 \\
\hline 6 & 20.87 & 50.81 & 28.32 \\
\hline \multicolumn{3}{|c|}{ Starting from $\{\mathbf{1 , 2}\}$} \\
\hline 1 & 20.22 & 51.73 & 28.06 \\
\hline 3 & 20.83 & 50.43 & 28.74 \\
\hline 4 & 22.38 & 50.16 & 27.46 \\
\hline 5 & 22.44 & 50.06 & 27.50 \\
\hline 6 & 21.56 & 50.63 & 27.81 \\
\hline
\end{tabular}

Table 8. $15 \%$ Cholesterol + DPPC

\begin{tabular}{|c|c|c|c|}
\hline \multirow{2}{*}{} & \multicolumn{3}{|c|}{ Mol \% of equilibrium product mixtures } \\
\cline { 2 - 4 } Time, hrs & $\{\mathbf{1 , 1}\}$ & $\mathbf{\{ 1 , 2}\}$ & $\{\mathbf{2 , 2}\}$ \\
\hline 1 & 23.19 & 49.51 & 27.30 \\
\hline 2 & 21.85 & 49.80 & 28.36 \\
\hline 3 & 22.16 & 49.18 & 28.66 \\
\hline 4 & 22.84 & 49.18 & 27.98 \\
\hline 5 & 21.90 & 49.57 & 28.54 \\
\hline 6 & 20.99 & 49.57 & 29.44 \\
\hline & \multicolumn{3}{|c|}{ Starting from $\{\mathbf{1 , 2}\}$} \\
\hline 1 & 20.49 & 49.55 & 29.96 \\
\hline 2 & 21.61 & 49.32 & 29.08 \\
\hline 3 & 23.39 & 49.69 & 26.92 \\
\hline 4 & 22.48 & 48.08 & 29.44 \\
\hline 5 & 21.57 & 48.73 & 29.70 \\
\hline 6 & 22.95 & 49.27 & 27.78 \\
\hline
\end{tabular}




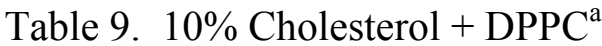

\begin{tabular}{|c|c|c|c|}
\hline \multirow{2}{*}{} & \multicolumn{3}{|c|}{ Mol \% of equilibrium product mixtures } \\
\cline { 2 - 4 } & \multicolumn{3}{|c|}{ Starting from $\{\mathbf{1 , 1}\}$ and $\{\mathbf{2 , 2}\}$} \\
\hline Time, hrs & $\{\mathbf{1}\}$ & $\{\mathbf{1 , 2}\}$ & $\{\mathbf{2 , 2}\}$ \\
\hline 1 & 23.61 & 49.33 & 27.06 \\
\hline 2 & 22.94 & 48.68 & 28.38 \\
\hline 3 & 22.39 & 48.90 & 28.70 \\
\hline 4 & 22.40 & 49.02 & 28.58 \\
\hline 5 & 22.05 & 48.99 & 28.96 \\
\hline 6 & 23.00 & 48.74 & 28.25 \\
\hline & \multicolumn{3}{|c|}{ Starting from $\{\mathbf{1 , 2}\}$} \\
\hline 1 & 22.26 & 49.51 & 28.23 \\
\hline 2 & 22.39 & 48.68 & 28.93 \\
\hline 3 & 22.03 & 49.20 & 28.78 \\
\hline 4 & 21.73 & 49.09 & 29.19 \\
\hline 5 & 23.07 & 48.89 & 28.04 \\
\hline 6 & 22.15 & 48.58 & 29.27 \\
\hline
\end{tabular}

${ }^{a}$ A small excess of $\{\mathbf{2}, \mathbf{2}\}$ relative to $\{\mathbf{1}, \mathbf{1}\}$ is observed only when low cholesterol concentrations were used. This is a likely consequence of higher amounts of thiol monomer present, when using low probe concentrations, and also because of differences in redox potentials, reflecting the sterol's greater tendendency to dimerize in the liquiddisordered state; that is, slightly more of $\mathbf{1}$ is present in its monomeric form than 2 . $^{5}$ Addition of cholesterol has a leveling effect by being able to form noncovalent dimers with thiol monomers of $\mathbf{2}$, thereby increasing their relative stability. It should noted that these dimer/monomer equilibria are completely independent of the dimer/dimer equilibria and do not, in any way, affect $K$ values.

Table 10. $10 \%$ Cholesterol + DPPC

\begin{tabular}{|c|c|c|c|}
\hline \multirow{2}{*}{ Time, hrs } & \multicolumn{3}{|c|}{ Mol \% of equilibrium product mixtures } \\
\cline { 2 - 4 } & $\{\mathbf{1 , 1}\}$ & $\{\mathbf{1 , 2}\}$ & $\{\mathbf{2 , 2}\}$ \\
\hline 1 & 22.73 & 48.79 & 28.48 \\
\hline 2 & 25.22 & 46.74 & 28.05 \\
\hline 3 & 26.00 & 43.41 & 30.60 \\
\hline 4 & 25.44 & 49.19 & 25.38 \\
\hline 5 & 24.70 & 48.95 & 26.34 \\
\hline 6 & 24.81 & 48.87 & 26.32 \\
\hline & \multicolumn{3}{|c|}{ Starting from $\{\mathbf{1 , 2}\}$} \\
\hline 1 & 19.68 & 49.54 & 30.79 \\
\hline 2 & 22.25 & 46.57 & 31.17 \\
\hline 3 & 21.45 & 48.32 & 30.22 \\
\hline 4 & 22.26 & 47.03 & 30.71 \\
\hline 5 & 23.87 & 46.13 & 30.00 \\
\hline 6 & 23.01 & 48.06 & 28.93 \\
\hline
\end{tabular}


Table 11. $40.0 \%$ Cholesterol + DSPC

\begin{tabular}{|c|c|c|c|}
\hline \multirow{2}{*}{} & \multicolumn{3}{|c|}{ Mol \% of equilibrium product mixtures } \\
\cline { 2 - 4 } & \multicolumn{3}{|c|}{ Starting from $\{\mathbf{2 , 2}\}$ and $\{\mathbf{3 , 3}\}$} \\
\hline Time, hrs & $\mathbf{3 , 3}\}$ & $\mathbf{2 , 3}\}$ & $\{\mathbf{2 , 2}\}$ \\
\hline 1 & 20.45 & 54.77 & 24.78 \\
\hline 2 & 19.02 & 55.05 & 25.93 \\
\hline 3 & 20.32 & 54.50 & 25.18 \\
\hline 4 & 20.26 & 54.66 & 25.08 \\
\hline 5 & 20.05 & 54.98 & 24.97 \\
\hline 6 & 20.40 & 54.80 & 24.80 \\
\hline \multicolumn{3}{|c|}{ Starting from $\{\mathbf{2 , 3}\}$} \\
\hline 1 & 19.84 & 54.52 & 25.64 \\
\hline 2 & 19.96 & 54.32 & 25.72 \\
\hline 3 & 21.46 & 51.84 & 26.69 \\
\hline 4 & 22.71 & 53.87 & 23.42 \\
\hline 5 & 20.72 & 54.91 & 24.37 \\
\hline 6 & 20.54 & 54.75 & 24.71 \\
\hline
\end{tabular}

Table 12. $35.0 \%$ Cholesterol + DSPC

\begin{tabular}{|c|c|c|c|}
\hline \multirow{2}{*}{} & \multicolumn{3}{|c|}{ Mol \% of equilibrium product mixtures } \\
\cline { 2 - 4 } & \multicolumn{3}{|c|}{ Starting from $\{\mathbf{2 , 2}\}$ and $\{\mathbf{3 , 3}\}$} \\
\hline Time, hrs & $\mathbf{3 , 3}\}$ & $\{\mathbf{2 , 3}\}$ & $\{\mathbf{2 , 2}\}$ \\
\hline 1 & 18.95 & 54.92 & 26.13 \\
\hline 2 & 19.57 & 54.92 & 25.52 \\
\hline 3 & 19.76 & 55.01 & 25.24 \\
\hline 4 & 20.07 & 55.11 & 24.82 \\
\hline 5 & 21.30 & 54.90 & 23.80 \\
\hline 6 & 19.74 & 53.83 & 26.43 \\
\hline \multicolumn{3}{|c|}{ Starting from $\{\mathbf{2 , 3}\}$} \\
\hline 1 & 20.13 & 55.03 & 24.83 \\
\hline 2 & 22.26 & 54.66 & 23.08 \\
\hline 3 & 20.45 & 54.24 & 25.30 \\
\hline 4 & 20.72 & 55.25 & 24.03 \\
\hline 5 & 19.19 & 55.11 & 25.70 \\
\hline 6 & 20.48 & 55.41 & 24.11 \\
\hline
\end{tabular}


Table 13. $30.0 \%$ Cholesterol + DSPC

\begin{tabular}{|c|c|c|c|}
\hline \multirow{2}{*}{} & \multicolumn{3}{|c|}{ Mol \% of equilibrium product mixtures } \\
\cline { 2 - 4 } & \multicolumn{3}{|c|}{ Starting from $\{\mathbf{3 , 3}\}$ and $\{\mathbf{2 , 2}\}$} \\
\hline Time, hrs & $\mathbf{3 , 3}\}$ & $\mathbf{2 , 3}\}$ & $\{\mathbf{2 , 2}\}$ \\
\hline 1 & 17.45 & 54.20 & 28.35 \\
\hline 2 & 18.14 & 54.11 & 27.75 \\
\hline 3 & 18.79 & 54.15 & 27.06 \\
\hline 4 & 21.52 & 50.31 & 28.17 \\
\hline 5 & 19.75 & 54.46 & 25.80 \\
\hline 6 & 17.30 & 51.92 & 30.78 \\
\hline \multicolumn{3}{|c|}{ Starting from $\{\mathbf{2 , 3}\}$} \\
\hline 1 & 18.09 & 56.03 & 25.88 \\
\hline 2 & 19.74 & 54.59 & 25.67 \\
\hline 3 & 20.14 & 54.72 & 25.14 \\
\hline 4 & 19.36 & 54.33 & 26.31 \\
\hline 5 & 18.30 & 55.29 & 26.41 \\
\hline 6 & 19.74 & 55.44 & 24.82 \\
\hline
\end{tabular}

Table 14. $27.5 \%$ Cholesterol + DSPC

\begin{tabular}{|c|c|c|c|}
\hline \multirow{2}{*}{} & \multicolumn{3}{|c|}{ Mol \% of equilibrium product mixtures } \\
\cline { 2 - 4 } & \multicolumn{3}{|c|}{ Starting from $\{\mathbf{3 , 3}\}$ and $\{\mathbf{2 , 2}\}$} \\
\hline Time, hrs & $\mathbf{3 , 3}\}$ & $\{\mathbf{2 , 3}\}$ & $\{\mathbf{2 , 2}\}$ \\
\hline 1 & 19.20 & 54.04 & 26.76 \\
\hline 2 & 18.39 & 53.87 & 27.74 \\
\hline 3 & 19.02 & 53.82 & 27.16 \\
\hline 4 & 18.62 & 54.64 & 26.74 \\
\hline 5 & 18.21 & 54.09 & 27.70 \\
\hline 6 & 18.85 & 54.17 & 26.98 \\
\hline \multicolumn{3}{|c|}{ Starting from $\{\mathbf{2 , 3}\}$} \\
\hline 1 & 19.36 & 54.04 & 26.60 \\
\hline 2 & 23.40 & 49.39 & 27.21 \\
\hline 3 & 21.29 & 53.41 & 25.30 \\
\hline 4 & 20.49 & 53.58 & 25.93 \\
\hline 5 & 21.00 & 53.66 & 25.34 \\
\hline 6 & 19.53 & 54.48 & 25.99 \\
\hline
\end{tabular}


Table 15. 25.0 \% Cholesterol + DSPC

\begin{tabular}{|c|c|c|c|}
\hline \multirow{2}{*}{} & \multicolumn{3}{|c|}{ Mol \% of equilibrium product mixtures } \\
\cline { 2 - 4 } & $\mathbf{3 , 3}\}$ & $\{\mathbf{2 , 3}\}$ & $\{\mathbf{2}\}$ and $\boldsymbol{\mathbf { 2 } , \mathbf { 2 } \}}$ \\
\hline Time, hrs & 20.00 & 52.02 & 27.99 \\
\hline 1 & 19.37 & 52.85 & 27.78 \\
\hline 2 & 18.84 & 53.13 & 28.03 \\
\hline 3 & 18.91 & 53.64 & 27.45 \\
\hline 4 & 19.11 & 54.01 & 26.88 \\
\hline 5 & 18.32 & 53.46 & 28.22 \\
\hline 6 & \multicolumn{3}{|c|}{ Starting from 18-Cho } \\
\hline \multicolumn{4}{|c|}{} \\
\hline 1 & 19.06 & 53.45 & 27.49 \\
\hline 2 & 19.97 & 54.08 & 25.95 \\
\hline 3 & 19.38 & 53.69 & 26.92 \\
\hline 4 & 19.37 & 53.65 & 26.98 \\
\hline 5 & 19.69 & 53.46 & 26.85 \\
\hline 6 & 19.52 & 54.06 & 26.42 \\
\hline
\end{tabular}

Table 16. $20.0 \%$ Cholesterol + DSPC

\begin{tabular}{|c|c|c|c|}
\hline \multirow{2}{*}{} & \multicolumn{3}{|c|}{ Mol \% of equilibrium product mixtures } \\
\cline { 2 - 4 } & $\mathbf{3 , 3}\}$ & $\mathbf{2}, \mathbf{3}\}$ & $\mathbf{3}\}$ and $\mathbf{2}, \mathbf{2}\}$ \\
\hline Time, hrs & 18.90 & 50.69 & 30.41 \\
\hline 1 & 20.41 & 52.12 & 27.47 \\
\hline 2 & 18.79 & 51.83 & 29.37 \\
\hline 4 & 17.88 & 52.44 & 29.68 \\
\hline 5 & 18.17 & 52.34 & 29.49 \\
\hline 6 & \multicolumn{3}{|c|}{ Starting from $\{\mathbf{2 , 3}\}$} \\
\hline \multicolumn{4}{|c|}{} \\
\hline 1 & 18.41 & 52.47 & 29.13 \\
\hline 3 & 19.08 & 52.40 & 28.52 \\
\hline 4 & 18.29 & 52.86 & 28.84 \\
\hline 5 & 19.34 & 52.32 & 28.34 \\
\hline 6 & 18.98 & 52.77 & 28.25 \\
\hline
\end{tabular}


Table 17. $15.0 \%$ Cholesterol + DSPC

\begin{tabular}{|c|c|c|c|}
\hline \multirow{2}{*}{} & \multicolumn{3}{|c|}{ Mol \% of equilibrium product mixtures } \\
\cline { 2 - 4 } & \multicolumn{3}{|c|}{ Starting from $\{\mathbf{3 , 3}\}$ and $\{\mathbf{2 , 2}\}$} \\
\hline Time, hrs & $\mathbf{3 3 , 3}\}$ & $\mathbf{2 , 3}\}$ & $\{\mathbf{2 , 2}\}$ \\
\hline 1 & 18.97 & 49.48 & 31.56 \\
\hline 2 & 20.66 & 49.32 & 30.02 \\
\hline 3 & 18.96 & 49.55 & 31.49 \\
\hline 4 & 17.37 & 49.37 & 33.25 \\
\hline 5 & 20.68 & 49.55 & 29.77 \\
\hline 6 & 17.15 & 49.58 & 33.27 \\
\hline \multicolumn{3}{|c|}{ Starting from $\{\mathbf{2 , 3}\}$} \\
\hline 1 & 19.15 & 52.30 & 28.55 \\
\hline 2 & 20.26 & 50.37 & 29.36 \\
\hline 3 & 21.26 & 50.79 & 27.95 \\
\hline 4 & 21.71 & 50.96 & 27.33 \\
\hline 5 & 20.12 & 48.34 & 31.54 \\
\hline 6 & 18.37 & 51.66 & 29.97 \\
\hline
\end{tabular}

Table 18. $12.5 \%$ Cholesterol + DSPC

\begin{tabular}{|c|c|c|c|}
\hline \multirow{2}{*}{} & \multicolumn{3}{|c|}{ Mol \% of equilibrium product mixtures } \\
\cline { 2 - 4 } & \multicolumn{3}{|c|}{ Starting from $\{\mathbf{3 , 3}\}$ and $\{\mathbf{2 , 2}\}$} \\
\hline Time, hrs & $\mathbf{3 , 3}\}$ & $\{\mathbf{2 , 3}\}$ & $\{\mathbf{2 , 2}\}$ \\
\hline 1 & 20.78 & 49.05 & 30.17 \\
\hline 2 & 20.56 & 49.35 & 30.09 \\
\hline 3 & 20.72 & 49.85 & 29.43 \\
\hline 4 & 20.79 & 49.48 & 29.73 \\
\hline 5 & 20.35 & 49.52 & 30.12 \\
\hline 6 & 19.83 & 49.07 & 31.10 \\
\hline \multicolumn{3}{|c|}{ Starting from $\{\mathbf{2 , 3}\}$} \\
\hline 1 & 20.53 & 50.18 & 29.29 \\
\hline 2 & 20.64 & 49.13 & 30.23 \\
\hline 3 & 18.87 & 49.53 & 31.60 \\
\hline 4 & 20.59 & 48.74 & 30.67 \\
\hline 5 & 20.51 & 48.90 & 30.58 \\
\hline 6 & 19.74 & 49.40 & 30.86 \\
\hline
\end{tabular}


Table 19. $10.0 \%$ Cholesterol + DSPC

\begin{tabular}{|c|c|c|c|}
\hline \multirow{2}{*}{} & \multicolumn{3}{|c|}{ Mol \% of equilibrium product mixtures } \\
\cline { 2 - 4 } Starting from $\{\mathbf{3 , 3}\}$ and $\{\mathbf{2 , 2}\}$ \\
\hline Time, hrs & $\mathbf{3 , 3}\}$ & $\{\mathbf{2 , 3}\}$ & $\{\mathbf{2 , 2}\}$ \\
\hline 1 & 19.26 & 48.17 & 32.57 \\
\hline 2 & 18.92 & 49.46 & 31.62 \\
\hline 3 & 18.48 & 48.55 & 32.97 \\
\hline 4 & 20.54 & 48.67 & 30.79 \\
\hline 5 & 20.84 & 47.58 & 31.58 \\
\hline 6 & 20.15 & 45.89 & 33.96 \\
\hline \multicolumn{3}{|c|}{ Starting from $\{\mathbf{2 , 3}\}$} \\
\hline 1 & 21.75 & 48.89 & 29.36 \\
\hline 2 & 21.14 & 47.83 & 31.04 \\
\hline 3 & 20.23 & 47.18 & 32.59 \\
\hline 4 & 21.49 & 48.80 & 29.71 \\
\hline 5 & 18.12 & 48.84 & 33.04 \\
\hline
\end{tabular}

Table 20. $5.0 \%$ Cholesterol + DSPC

\begin{tabular}{|c|c|c|c|}
\hline \multirow{2}{*}{} & \multicolumn{3}{|c|}{ Mol \% of equilibrium product mixtures } \\
\cline { 2 - 4 } & \multicolumn{3}{|c|}{ Starting from $\{\mathbf{3 , 3}\}$ and $\{\mathbf{2 , 2}\}$} \\
\hline Time, hrs & $\mathbf{3 3 , 3}\}$ & $\{\mathbf{2 , 3}\}$ & $\{\mathbf{2 , 2}\}$ \\
\hline 1 & 20.54 & 45.12 & 34.33 \\
\hline 2 & 21.99 & 43.07 & 34.94 \\
\hline 3 & 19.36 & 47.15 & 33.50 \\
\hline 4 & 18.66 & 43.45 & 37.90 \\
\hline 5 & 21.05 & 47.59 & 31.36 \\
\hline 6 & 19.18 & 47.50 & 33.32 \\
\hline \multicolumn{3}{|c|}{ Starting from $\{\mathbf{2 , 3}\}$} \\
\hline 1 & 18.48 & 47.32 & 34.20 \\
\hline 2 & 19.52 & 47.53 & 32.95 \\
\hline 3 & 18.60 & 48.01 & 33.40 \\
\hline 4 & 18.10 & 47.33 & 34.57 \\
\hline 5 & 18.75 & 47.51 & 33.74 \\
\hline 6 & 18.99 & 47.27 & 33.74 \\
\hline
\end{tabular}

